\title{
EQUIVARIANT DIFFERENTIAL CHARACTERS AND SYMPLECTIC REDUCTION
}

\author{
EUGENE LERMAN AND ANTON MALKIN
}

\begin{abstract}
We describe equivariant differential characters (classifying equivariant circle bundles with connections), their prequantization, and reduction.
\end{abstract}

\section{Contents}

0. Introduction

0.1. Prequantization

0.2. Lie group actions

0.3 . The stack of bundles and the stack of differential characters

0.4. Invariant connections

0.5. Symplectic reduction

0.6. General Lie groupoids

0.7. Structure of the paper

0.8. Previous Results

0.9. Higher degree versions

1. Circle bundles with connections and prequantization

1.1. Prequantization

1.2. Equivariant $S^{1}$-bundles on groupoids

1.3. Connections on equivariant $S^{1}$-bundles

1.4. Singular and de Rham cohomology of groupoids

1.5. Differential characters on groupoids

1.6. Equivariant Kostant Theorem

2. Invariant connections and the moment map

2.1. The moment map

2.2. Invariant connections

2.3. Prequantization on global quotients

3. Reduction

3.1. General Reduction

3.2. Reduction for action groupoids

4. Differential Characters as Characters

4.1. Differential characters on manifolds and groupoids 17

4.2. Special cases

4.3. Invariant differential characters on an action groupoid 20

5. Examples

5.1. Example: classifying stack

5.2. Example: coadjoint orbits of compact Lie groups

Supported in part by NSF grant DMS-0603892.

Supported in part by NSF grant DMS-0456714. 
5.3. Example: coadjoint orbits of $S U(2)$ and $S^{1}$-reduction 22 References

\section{INTRODUCTION}

This paper is a continuation of [10. The goal of the project is to understand geometric (pre)quantization and symplectic reduction in the context of stacks. There are two practical reasons for doing that. First, even if one is only interested in global Lie group actions on manifolds, symplectic quotients are generally orbifolds (i.e. Deligne-Mumford stacks). Second, the language of stacks allows one to work locally and thus to avoid messy Čech-type arguments.

0.1. Prequantization. The classic geometric prequantization theorem (due to Weil [15] and Kostant 9]) says that given a closed integral differential 2-form $\omega$ on a manifold $M$ there exists a principal $S^{1}$-bundle $P \rightarrow M$ with a connection $A$ such that the curvature of $A$ is equal to $\omega$, and moreover, the set of isomorphism classes of such pairs $(P, A)$ is a principal homogeneous space of the group of flat $S^{1}$-bundles. Recall that a 2 -form $\omega$ on a manifold $M$ is integral if $\int_{S} \omega \in \mathbb{Z}$ for any closed smooth singular 2-chain $S \in Z_{2}(M)$.

It would be preferable for prequantization procedure to produce unique output (an $S^{1}$-bundle with connection). For example, this is clearly required to make sense of statements like "prequantization commutes with reduction" - see below. In order to have unique output of the prequantization one has to refine its input. One way to do it, due to Cheeger and Simons [5], is by using differential characters (an alternative approach is provided by Deligne cohomology - see [3]). A differential character of degree 2 (degree 1 in Cheeger-Simons grading) is a pair $(\omega, \chi)$, where $\omega \in \Omega^{2}(M)$ is a closed 2-form and $\chi: Z_{1}(M) \rightarrow \mathbb{R} / \mathbb{Z}$ is a character of the group $Z_{1}(M)$ of smooth singular 1-cycles. This pair should satisfy the following compatibility condition:

$$
\chi(\partial S)=\int_{S} \omega \bmod \mathbb{Z},
$$

for any smooth singular 2-chain $S \in C_{2}(M)$. One can show (cf. [5,8, 10, for various versions of the proof) that differential characters classify isomorphism classes of principal $S^{1}$-bundles with connections. The classifying bijection (whose inverse is the prequantization map) associates to a principal $S^{1}$-bundle $P$ with a connection $A$ its differential character $(\omega, \chi)$, where $\omega$ is the curvature of $A$ and $\chi$ is the holonomy of $A$ (we identify $S^{1}$ with $\mathbb{R} / \mathbb{Z}$ throughout the paper).

0.2 . Lie group actions. Let $G$ be a Lie group, $\mathfrak{g}$ the Lie algebra of $G$, $\mathfrak{g}^{*}$ the dual space of $\mathfrak{g}$, and $<,>$ the natural pairing between $\mathfrak{g}$ and $\mathfrak{g}^{*}$. Suppose $G$ acts on a manifold $M$ and consider a $G$-equivariant principal $S^{1}$-bundle $P \rightarrow M$ with an invariant connection $A$. That means we are given a lifting of the $G$-action to the total space $P$ (commuting with the $S^{1}$-action) and the connection form $A \in \Omega^{1}(P)$ is $G$-invariant. Then the curvature $\omega$ of $A$ is also $G$-invariant and moreover there is a $G$-equivariant map $\mu: P \rightarrow \mathfrak{g}^{*}$ given by $\langle\mu, X\rangle=i_{\varepsilon_{x}} A$, where $\varepsilon_{X}$ is the vector field on $P$ generating the action of an element $X \in \mathfrak{g}$. The map $\mu$ is $S^{1}$ invariant and hence descends to a map from $M$ to $\mathfrak{g}^{*}$ which we also denote by $\mu$. It follows from the definition of the curvature that $i_{\varepsilon_{x}} \omega=-\langle d \mu, X>$. Hence $\mu$ is 
the moment map in the sense of equivariant symplectic geometry (see for example 7]). Note that if $G$-action is free (so that the quotient $M / G$ is a manifold) then a $G$-equivariant bundle $P$ with an invariant connection $A$ descends to $M / G$ iff the moment map is identically equal to zero, in other words iff the forms $A$ and $\omega$ are $G$ basic. Recall that a differential form is $G$-basic if it is $G$-invariant and $G$-horizontal (i.e. vanish on vector fields generating the action of $\mathfrak{g}$ ).

Equivariant prequantization procedure should produce a $G$-equivariant principal $S^{1}$-bundle with an invariant connection given a closed invariant integral 2-form $\omega$ on $M$. Obviously there some obstructions. First of all there should exist a moment map $\mu$ such that $i_{\varepsilon_{x}} \omega=-\langle d \mu, X>$ for any $X \in \mathfrak{g}$. This means that the action is Hamiltonian. If $G$ is connected and simply-connected then existence of the moment map ensures existence of an equivariant prequantization bundle with an invariant connection (cf. 9]). For a general $G$ there are additional obstructions. The origin of these complications is that in the equivariant situation it is not enough to think of isomorphism classes of bundles - one needs to consider an actual bundle in order to lift the $G$-action to the total space. This becomes much more clear if one uses an equivalent definition of an equivariant bundle in terms of the action groupoid $M \underset{p}{\longleftarrow} G \times M$, where the two maps are the projection and the action. In this language an equivariant $S^{1}$-bundle is a bundle $P \rightarrow M$ together with an isomorphism $\varphi: a^{*} P \stackrel{\sim}{\rightarrow} p^{*} P$ of the two pull-backs of $P$ to $G \times M$. Note that we have to use a morphism in the category of bundles on $G \times M$, so it is not enough to consider isomorphism classes of bundles. Therefore in order to understand equivariant prequantization, we have to upgrade the prequantization map to a functor from some category of differential characters to the category of principal $S^{1}$-bundles with connections.

0.3 . The stack of bundles and the stack of differential characters. Let $\mathcal{D B S}^{1}(M)$ be the category of $S^{1}$-bundles with connections on a manifold $M$. In 8 Hopkins and Singer introduced a category of differential characters $\mathcal{D} \mathcal{C}_{2}^{2}(M)$ and showed that it is equivalent to $\mathcal{D B} \mathcal{S}^{1}(M)$. As the name suggests, the isomorphism classes of objects of $\mathcal{D C}_{2}^{2}(M)$ are in bijection with differential characters. We give the complete definition of the category $\mathcal{D C}_{2}^{2}(M)$ in Subsection 1.1. Let us just mention that an object of $\mathcal{D C}_{2}^{2}(M)$ is a triple $(c, h, \omega)$, where $c \in Z^{2}(M, \mathbb{Z})$ is a smooth singular 2-cocycle, $\omega \in \Omega^{2}(M)$ is a closed 2-form, and $h \in C^{1}(M, \mathbb{R})$ is a smooth singular 1-chain satisfying $d h=c-\omega$. In the previous paper [10] we introduced a prequantization functor

$$
\text { Preq : } \mathcal{D C}_{2}^{2}(M) \rightarrow \mathcal{D B S}^{1}(M)
$$

such that if $\operatorname{Preq}((c, h, \omega))$ is a bundle $P$ with a connection $A$, then the Chern class of $P$ is $[c] \in H^{2}(M, \mathbb{Z})$ and the curvature of $A$ is $\omega$. Moreover we show (and it is crucial for our definition of Preq) that both $\mathcal{D C}_{2}^{2}$ and $\mathcal{D B S}{ }^{1}$ are stacks over the category of manifolds and Preq is an equivalence of stacks. This allowed us to define geometric prequantization of arbitrary stacks over the category of manifolds, in particular prequantization of orbifolds and equivariant prequantization of manifolds with Lie group actions. For example, given a manifold $M$ with an action of a Lie group $G$ we have an equivalence functor

$$
\mathcal{D C}_{2}^{2}(M \leftleftarrows G \times M) \simeq \mathcal{D C}_{2}^{2}([M / G]) \stackrel{\text { Preq }}{\longrightarrow} \mathcal{D B S}^{1}([M / G]) \simeq \mathcal{D B S}^{1}(M \leftleftarrows G \times M)
$$


Here $[M / G]$ is the quotient stack. Objects of the category $\mathcal{D B S} \mathcal{S}^{1}(M \leftleftarrows G \times M)$ are $G$-equivariant principal $S^{1}$-bundles on $M$ with $G$-basic connections. The category $\mathcal{D C}_{2}^{2}(M \leftleftarrows G \times M)$ of equivariant differential characters is defined in Section 1.

0.4. Invariant connections. In the present paper we are interested in equivariant bundles with $G$-invariant (but not necessarily basic) connections. These form a category $d \mathcal{B S}_{\text {inv }}^{1}(M \leftleftarrows G \times M)$. The corresponding category of differential characters is defined in Section 2 and is denoted by $\mathcal{D C}_{\text {inv }}^{2}(M \leftleftarrows G \times M)$. In particular a $G$-invariant differential character (i.e. an isomorphism class of objects of the category $\left.\mathcal{D C}_{\text {inv }}^{2}(M \leftleftarrows G \times M)\right)$ is a triple $(\omega, \mu, \Psi)$, where $\omega \in \Omega^{2}(M)$ is a 2-form, $\mu: M \rightarrow \mathfrak{g}^{*}$ is a (moment) map, and $\Psi$ is a character $Z_{1}(M \leftleftarrows G \times M) \rightarrow \mathbb{R} / \mathbb{Z}$ of the group $Z_{1}(M \leftleftarrows G \times M)$ of smooth singular 1-cycles on the action groupoid. We refer the reader to Section 4 for details, but let us give here two examples of subgroups of $Z_{1}(M \leftleftarrows G \times M)$. First one is the group $Z_{1}(M)$ of 1-cycles on $M$. The restriction of $\Psi$ to $Z_{1}(M)$ together with $\omega$ form a differential character on $M$ corresponding to a bundle with connection (without equivariant structure). The second typical subgroup of $Z_{1}(M \leftleftarrows G \times M)$ is the stabilizer group (in $\left.G\right)$ of a point on $M$. The restriction of $\Psi$ to this subgroup corresponds (under prequantization map) to the action of the stabilizer group on the fiber of the prequantization $S^{1}$ bundle. Let us also note that the character is equivariant (in other words, belongs to $\mathcal{D C}_{2}^{2}(M \leftleftarrows G \times M) \simeq \mathcal{D} \mathcal{C}_{2}^{2}([M / G])$ iff $\omega$ is basic or, equivalently, iff $\mu$ vanishes identically.

Here is the first main result of the present paper stated both in terms of categories and isomorphism classes of objects (see Theorems 2.3.1 and 4.3.1 for more precise/complete statements) .

Theorem 0.4.1. Suppose a Lie group $G$ acts on a manifold $M$.

- There is an equivalence of categories (prequantization functor)

$$
\text { Preq : } \mathcal{D C}_{\text {inv }}^{2}(M \leftleftarrows G \times M) \stackrel{\sim}{\rightarrow} d \mathcal{B S}_{\text {inv }}^{1}(M \leftleftarrows G \times M)
$$

from the category $\mathcal{D C}_{\text {inv }}^{2}(M \leftleftarrows G \times M)$ of $G$-invariant differential characters to the category $d \mathcal{B} \mathcal{S}_{\text {inv }}^{1}(M \leftleftarrows G \times M)$ of $G$-equivariant principal $S^{1}$-bundles with $G$-invariant connections.

- Isomorphism classes of $G$-equivariant $S^{1}$-bundles with $G$-invariant connections are in bijection with $G$-invariant differential characters, i.e. triples $(\omega, \mu, \Psi)$, where $\omega \in \Omega^{2}(M)$ is a G-invariant 2-form (the curvature of the connection), $\mu: M \rightarrow \mathfrak{g}^{*}$ is a $G$-equivariant (moment) map, and $\Psi$ is a $G$-invariant character $Z_{1}(M \leftleftarrows G \times M) \rightarrow \mathbb{R} / \mathbb{Z}$.

0.5. Symplectic reduction. We continue with our setup of a Lie group $G$ acting on a manifold $M$, a $G$-equivariant principal $S^{1}$-bundle $P \rightarrow M$ with a $G$-invariant connection $A \in \Omega^{1}(P)$, the curvature $\omega \in \Omega^{1}(M)$, and the moment map $\mu: M \rightarrow$ $\mathfrak{g}^{*}$. Assume zero is a regular value of the moment map. Then $\mu^{-1}(0)$ is a manifold and the restriction of the pair $(P, A)$ to $\mu^{-1}(0)$ is a principal $S^{1}$-bundle with a basic connection, hence a bundle with connection on the stack $\left[\mu^{-1}(0) / G\right]$. On the other hand one can restrict the corresponding differential character $(\omega, \mu, \Psi)$ to $\mu^{-1}(0)$ and the restricted character descends to the quotient stack $\left[\mu^{-1}(0) / G\right]$. In particular the form $\left.\omega\right|_{\mu^{-1}(0)}$ descends to $\left[\mu^{-1}(0) / G\right]$, which is the point of symplectic reduction (to be honest, a deeper statement is about non-degeneracy of the reduction of a 
non-degenerate 2-form $\omega$ [12,13, but the non-degeneracy condition is not relevant for geometric (pre)quantization).

Our second result is that the prequantization bijection between bundles with connections and differential characters commutes with reduction. Since we are interested in equivalences of categories let us change the point of view and consider all bundles/characters such that their moment map vanishes on a given $G$-stable submanifold $N \subset M$. We denote the corresponding subcategories by $d \mathcal{B S}_{\text {inv }, N}^{1}(M \leftleftarrows G \times M)$ and $\mathcal{D C}_{\text {inv }, N}^{2}(M \leftleftarrows G \times M)$. Then we have the following result (see Theorem 3.2.1).

Theorem 0.5.1. Let a Lie group $G$ act on a manifold $M$ and $i: N \hookrightarrow M$ an inclusion of a $G$-stable submanifold. The diagram

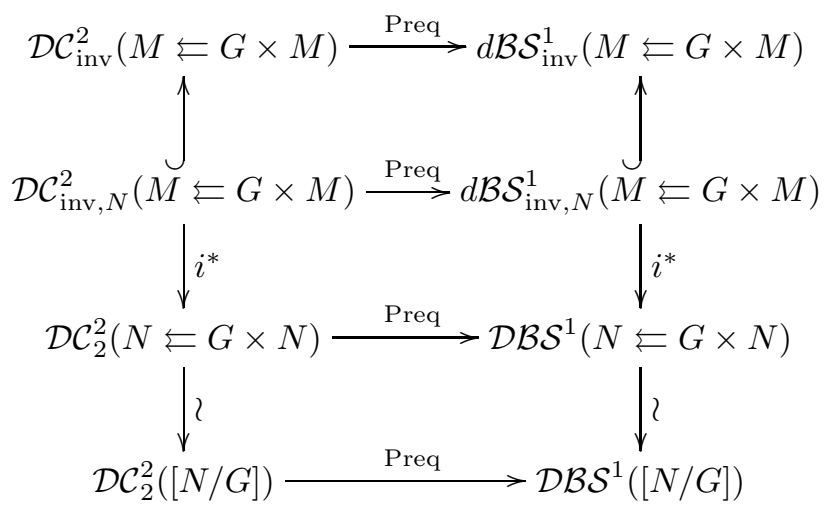

commutes up to natural transformations. Here the first row represents $G$-invariant characters/connections, the second row has moments vanishing on the submanifold $N$, the third is the restriction of the second to $N$, and the fourth is the descent of the third to the quotient (which makes sense because of the moment vanishing).

0.6. General Lie groupoids. The above discussion concerned bundles, connections, and differential characters, in the setup of a Lie group $G$ acting on a manifold $M$. However it should be clear by now that definitions and results are best stated using the language of an action groupoid $M \leftleftarrows G \times M$. Hence it makes sense to work in the context of a general Lie groupoid $M \leftleftarrows \Gamma$, which we do most of the time in the present paper. Our other motivation for using general groupoids is in that they provide atlases for stacks (and in particular for manifolds). In other words, we try to describe prequantization/reduction locally. One should be careful however with stacky interpretations of groupoid results. Not every constructions is atlas-independent (i.e. makes sense for the underlying stack). For example, basic connections descend to the quotient stack while invariant ones don't.

0.7. Structure of the paper. Our goal is to describe equivariant bundles with invariant connections in terms of differential characters. We do this in two steps. First, in Section 1, we define a category $d \mathcal{B S}^{1}(M \leftleftarrows \Gamma)$ of equivariant principal $S^{1}$ bundles with arbitrary connection. Roughly speaking, this category sits in between the category $\mathcal{B S}^{1}(M \leftleftarrows \Gamma)$ of equivariant bundles without connections and the category $\mathcal{D B S}^{1}(M \leftleftarrows \Gamma)$ of equivariant bundles with basic connections. Then in Theorem 1.5.1 we construct a (prequantizaion) equivalence

$$
\text { Preq : } \mathcal{D C}_{2-1}^{2}(M \leftleftarrows \Gamma) \stackrel{\sim}{\rightarrow} d \mathcal{B S}^{1}(M \leftleftarrows \Gamma)
$$


where $\mathcal{D C}_{2-1}^{2}(M \leftleftarrows \Gamma)$ is certain category of differential characters sitting between the category $\mathcal{D C}_{1}^{2}(M \leftleftarrows \Gamma)$ classifying equivariant principal $S^{1}$-bundles and the category $\mathcal{D C}_{2}^{2}(M \leftleftarrows \Gamma)$ classifying equivariant bundles with basic connections. This is the main technical result of the paper. Our construction of the functor Preq has two important features. First, it is explicit enough to allow us to track invariance properties of the connections via the corresponding differential characters. More precisely, a connection on an equivariant bundle canonically determines a 1 -form $\alpha \in \Omega^{1}(\Gamma)$ and this form is a part of the differential character (see Theorem 1.5.1 for details). Second property of Preq is that it commutes (up to natural transformations) with pull-backs (in particular, restrictions). This implies the "prequantization commutes with reduction" theorem.

On the second step, in Section 3, we translate invariance property of connections into conditions on the corresponding differential character and thus prove the first part of Theorem 0.4.1. We also discuss the moment map and invariant connections/curvatures on a general groupoid. In particular, we observe that the invariance condition only makes sense on the zero level of the moment map. So in the groupoid case the usual (global action) reduction procedure: consider invariant symplectic forms - restrict to the zero level of the moment map - take quotient, should be reversed as follows: restrict to the zero level of the moment map - consider invariant forms - take quotient (in fact, the quotient is a tautology in the stacky language).

In Section 3 we discuss reduction and prove Theorem 0.5.1.

In Section 4 we provide an explicit description of the actual differential characters on a groupoid (i.e of the isomorphism classes of objects of $\mathcal{D C _ { 2 - 1 } ^ { 2 }}(M \leftleftarrows \Gamma)$ ) and prove the second part of Theorem 0.4.1.

Finally, Section 5 provides some examples of differential characters and reduction.

0.8. Previous Results. There are several recent papers dealing with equivariant geometric prequantization and reduction. In the case of a global Lie group action Gomi [6] describes prequantization via equivariant Deligne cohomology. Lupercio and Uribe [11] describe equivariant differential characters for a finite group action (their construction is different from ours). Behrend and $\mathrm{Xu} 2$ prove a version of Kostant's prequantization theorem on proper groupoids (we explain how to derive a generalization of their result using differential characters in subsection 1.6).

Besides being restricted to global quotients (except [2]) the above papers deal with isomorphism classes rather than stacks of bundles. As explained above we believe that it is impossible to properly understand geometric quantization on stacks without working with categories of bundles (i.e. with both objects and morphisms).

Bos 4 deals with symplectic reduction in a Lie groupoid case. His approach (and in fact his problem) is different from ours - he studies certain internal symplectic structure/reduction.

As always when talking about symplectic structures and groupoids one should not confuse symplectic structures on the manifolds of objects (our situation) and on arrows ("symplectic groupoids" of Weinstein [16]).

0.9. Higher degree versions. In this paper we are dealing with invariant differential characters of degree 2. Similar results hold for degree 3 characters (classifying 
$S^{1}$-gerbes with connective structures). In particular equivariant string connections appear naturally in this approach.

We wanted to keep this text free of obscure notions of higher stacks and their descent conditions and so postponed discussion of gerbes and their characters for another paper.

\section{Circle bundles with connections and prequantization}

1.1. Prequantization. We recall the construction of the prequantization functor in [10. Let $M$ be a differentiable manifold and consider the category $\mathcal{D B S}^{1}(M)$ of principal $S^{1}$-bundles with connections on $M$, i.e. the objects of $\mathcal{D B S} \mathcal{S}^{1}(M)$ are $S^{1}$-bundles with connections and morphisms are smooth $S^{1}$-equivariant maps preserving connections. In [10] we described a prequantization functor

$$
\text { Preq : } \mathcal{D C}_{2}^{2}(M) \rightarrow \mathcal{D B S}^{1}(M)
$$

where $\mathcal{D C}_{2}^{2}(M)$ is the category of differential characters defined below. We also showed that Preq is an equivalence of categories. Thus one can say that differential characters classify $S^{1}$-bundles with connections.

To define the category of differential characters (following Hopkins-Singer 8] ) we start with a complex $D C_{s}^{\bullet}(M)$ of abelian groups:

$$
D C_{s}^{n}(M)=\{(c, h, \omega) \mid \omega=0 \text { if } n<s\} \subset C^{n}(M, \mathbb{Z}) \times C^{n-1}(M, \mathbb{R}) \times \Omega^{n}(M),
$$

where $C^{n}(M, R)$ denotes the group of smooth singular cochains with coefficients in a ring $R, \Omega^{n}(M)$ denotes the group of differential forms, and $s$ is a fixed positive integer (truncation degree). The differential in the complex $D C_{s}^{\bullet}(M)$ is given by

$$
d(c, h, \omega)=(d c, \omega-c-d h, d \omega) .
$$

The group of Cheeger-Simons differential characters $[5$ of degree 2 on $M$ is isomorphic to $H^{2}\left(D C_{2}^{\bullet}(M)\right)$.

The category $\mathcal{D C} \mathcal{C}_{2}^{2}(M)$ is defined as follows:

- Objects are 2-cocycles: $(c, h, \omega) \in D C_{2}^{2}(M), d(c, h, \omega)=0$

- Morphisms are 1-cochains up to exact 1-cochains. The set of morphisms from $(c, h, \omega)$ to $\left(c^{\prime}, h^{\prime}, \omega^{\prime}\right)$ is

$$
\frac{\left\{(a, t, 0) \in D C_{2}^{1}(M) \mid d(a, t, 0)=(c, h, \omega)-\left(c^{\prime}, h^{\prime}, \omega^{\prime}\right)\right\}}{(a, t, 0) \sim(a, t, 0)+d(m, 0,0) \text { for }(m, 0,0) \in D C_{2}^{0}(M)} .
$$

- Composition of morphisms is the addition in $D C_{2}^{1}(M)$.

Note that given a smooth map $\rho: M \rightarrow N$, one can define natural pull-back functors $\mathcal{D C}_{2}^{2}(\rho): \mathcal{D C}_{2}^{2}(N) \rightarrow \mathcal{D C}_{2}^{2}(M)$ and $\mathcal{D B S}^{1}(\rho): \mathcal{D B S}^{1}(N) \rightarrow \mathcal{D B S}^{1}(M)$. This means that $\mathcal{D} \mathcal{C}_{2}^{2}$ and $\mathcal{D B S} \mathcal{S}^{1}$ form presheaves of groupoids over the category of manifolds, and it is shown in [10] that these presheaves are in fact stacks. We don't use stacky language in the present paper but it is needed for the proof of the prequantization theorem below.

Now we are ready to define the prequantization functor. Let $\mathcal{D B S}_{\text {triv }}^{1}(M)$ denote the full subcategory of $\mathcal{D B S} \mathcal{S}^{1}(M)$ consisting of trivial bundles with arbitrary connections and consider a functor $\mathrm{DCh}_{\text {triv }}: \mathcal{D} \mathcal{B} \mathcal{S}_{\text {triv }}^{1} \rightarrow \mathcal{D C}_{2}^{2}$ given by:

- $\mathrm{DCh}_{\text {triv }}\left(\left(M \times S^{1}, \beta+d \theta\right)\right)=(0, \beta, d \beta)$ on objects. Here $\beta \in \Omega^{1}(M)$, $\theta \in \mathbb{R} / \mathbb{Z}$ is the coordinate on $S^{1}$, and $\beta+d \theta$ is a connection 1 -form on the trivial bundle $M \times S^{1} \rightarrow M$. 
- $\mathrm{DCh}_{\text {triv }}(f)=[(d(\tilde{f}-f),-\tilde{f}, 0)] \in D C_{2}^{1} / d\left(D C_{2}^{0}\right)$ on morphisms. Here we think of a morphism in $\mathcal{D} \mathcal{B} \mathcal{S}_{\text {triv }}^{1}$ from $\left(M \times S^{1}, a+d \theta\right)$ to $\left(M \times S^{1}, a^{\prime}+\right.$ $d \theta)$ as a smooth function $f: M \rightarrow S^{1}=\mathbb{R} / \mathbb{Z}$ such that $d f=a^{\prime}-a$ and let $\tilde{f} \in C^{0}(M, \mathbb{R})$ be a lift of $f$.

One of the main results of [10] is:

Theorem 1.1.1. There is unique (up to a natural transformation) equivalence of stacks

$$
\mathrm{DCh}: \mathcal{D B S}^{1} \rightarrow \mathcal{D C}_{2}^{2}
$$

extending $\mathrm{DCh}_{\text {triv }}$. In other words, there is a family of equivalence functors (one for each manifold):

$$
\operatorname{DCh}(M): \mathcal{D B S}^{1}(M) \rightarrow \mathcal{D C}_{2}^{2}(M)
$$

such that (i) DCh commutes (up to coherent natural transformations) with pullbacks, and (ii) on trivial bundles with connections $\mathrm{DCh}$ is equal to $\mathrm{DCh}_{\text {triv }}$.

We call a quasi-inverse functor of $\mathrm{DCh}$ prequantization functor and denote it Preq (it is defined up to a natural transformation).

The idea behind this theorem is that any bundle becomes trivial after pullback to a contractible open subset of $M$. A similar argument (cf. [10]) shows the category $\mathcal{D C}_{1}^{2}(M)$ is equivalent to the category $\mathcal{B S}^{1}$ of principal $S^{1}$-bundles (without connections).

1.2. Equivariant $S^{1}$-bundles on groupoids. Consider a Lie groupoid $M \leftleftarrows \Gamma$. Its nerve is the simplicial manifold

$$
M \underset{\partial_{0}=s}{\stackrel{\partial_{1}=t}{\longleftarrow}} \Gamma \underset{\partial_{0}}{\stackrel{\partial_{2}}{\leftrightarrows}} \Gamma_{2} \underset{\partial_{0}}{\stackrel{\partial_{3}}{\leftrightarrows}} \ldots
$$

where

$$
\Gamma_{n}=\underbrace{\Gamma \times_{M} \Gamma \times_{M} \ldots \times_{M} \Gamma}_{n} .
$$

The maps $\partial_{0}=s, \partial_{1}=t: \Gamma \rightarrow M$ are the source and the target maps of the groupoid respectively. We use notations $(s, t)$ and $\left(\partial_{0}, \partial_{1}\right)$ interchangeably throughout the paper. The maps $\partial_{0}, \partial_{n}: \Gamma_{n} \rightarrow \Gamma_{n-1}$ forget the first and the last $\Gamma$-factors respectively, and finally, $\partial_{i}: \Gamma_{n} \rightarrow \Gamma_{n-1}$ for $1 \leq i \leq n-1$ multiplies (composes) $i^{t h}$ and $(i+1)^{t h}$ factors. We omit face maps $\Gamma_{n-1} \rightarrow \Gamma_{n}$ (in particular the unit map for the groupoid) in the above diagram.

A principal $S^{1}$-bundle $P \rightarrow M$ is $\Gamma$-equivariant if one can lift it to a simplicial principal $S^{1}$-bundle

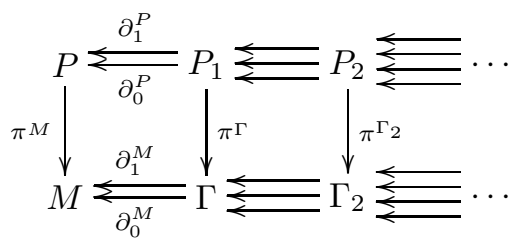

More precisely, such a lift provides an equivariant structure on the bundle. An equivalent definition of an equivariant structure on $P$ is a bundle isomorphism $\varphi:\left(\partial_{1}^{M}\right)^{*} P \stackrel{\sim}{\rightarrow}\left(\partial_{0}^{M}\right)^{*} P$ satisfying a cocycle condition on $\Gamma_{2}$. In the case of an 
action groupoid $M \leftleftarrows G \times M$ an equivariant structure on a bundle $P \rightarrow M$ is equivalent to a lift of the $G$-action on $\mathrm{M}$ to an action on the total space $P$.

1.3. Connections on equivariant $S^{1}$-bundles. Now suppose we are given a connection $A$ on the bundle $P \rightarrow M$. We think of $A$ as a 1 -form on $P$. The connection $A$ is $\Gamma$-basic (with respect to a given equivariant structure on $P$ ) if $\left(\partial_{1}^{P}\right)^{*} A=\left(\partial_{0}^{P}\right)^{*} A$. In the case of an action groupoid $M \leftleftarrows G \times M$ a connection is basic if it is $G$-invariant and vanish on vector fields generating the infinitesimal action of the Lie algebra $\mathfrak{g}$ of $G$. Equivariant $S^{1}$-bundles with basic connections form a category $\mathcal{D B S}^{1}(M \leftleftarrows \Gamma)$ with morphisms being equivariant bundle maps preserving connections. In fact (cf. [10) this category (up to an equivalence) depends only on the stack $[M / \Gamma]$, not on the actual (atlas) groupoid $M \leftleftarrows \Gamma$. In other words, it is Morita-equivariant.

In the present paper we are interested in arbitrary (not necessarily basic) connections on an equivariant $S^{1}$-bundle. So we introduce a category $d \mathcal{B S}^{1}(M \leftleftarrows \Gamma)$ of $\Gamma$ equivariant bundles on $M$ with arbitrary connections. Morphisms in $d \mathcal{B S}^{1}(M \leftleftarrows \Gamma)$ are equivariant bundle maps preserving connections. Note that $\mathcal{D B S}^{1}(M \leftleftarrows \Gamma)$ is a full subcategory of $d \mathcal{B S}^{1}(M \leftleftarrows \Gamma)$.

1.4. Singular and de Rham cohomology of groupoids. Let us recall the definitions of singular and de Rham complexes on a groupoid $M \leftleftarrows \Gamma$. We refer the reader to [1] for details. The de Rham complex $\Omega^{\bullet}(M \leftleftarrows \Gamma)$ is the total complex of the following double complex (denoted by $\Omega^{\bullet}\left(\Gamma_{\bullet}\right)$ ) :

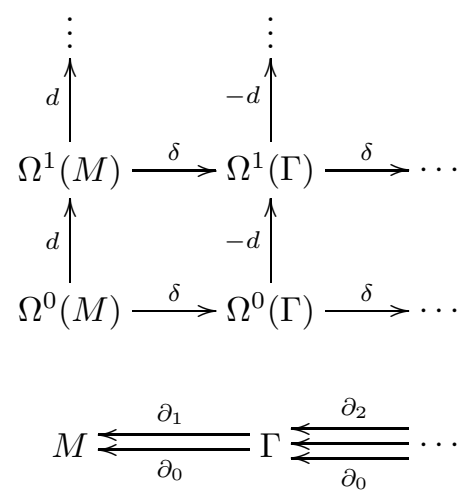

where $\delta$ is the simplicial differential: $\delta=\sum(-1)^{i} \partial_{i}^{*}$. Analogously, given a ring $R$, the smooth singular cochain complex $C^{\bullet}(M \leftleftarrows \Gamma, R)$ is defined as the total complex of the double complex $C^{\bullet}\left(\Gamma_{\bullet}, R\right)$. One can also define singular chains complex in a similar fashion (with arrows in the opposite direction). Smooth singular chains are dual to smooth singular cochains. The usual de Rham Theorem on manifolds implies (by a spectral sequence argument) de Rham Theorem on groupoids: $H^{n}\left(C^{\bullet}(M \leftleftarrows \Gamma, \mathbb{R})\right)$ is isomorphic to $H^{n}\left(\Omega^{\bullet}(M \leftleftarrows \Gamma)\right)$. The isomorphism is provided by integrating forms over smooth chains.

1.5. Differential characters on groupoids. Similarly to singular and de Rham cochain complexes on a groupoid $M \leftleftarrows \Gamma$ one can define the complex of differential characters $D C_{s}^{\bullet}(M \leftleftarrows \Gamma)$ as the total complex of the double complex $D C_{s}^{\bullet}\left(\Gamma_{\bullet}\right)$. It is shown in [10] that, provided $s>0$, the total cohomology of $D C_{s}^{\bullet}\left(\Gamma_{\bullet}\right)$ is equal to the total cohomology of its truncated version 


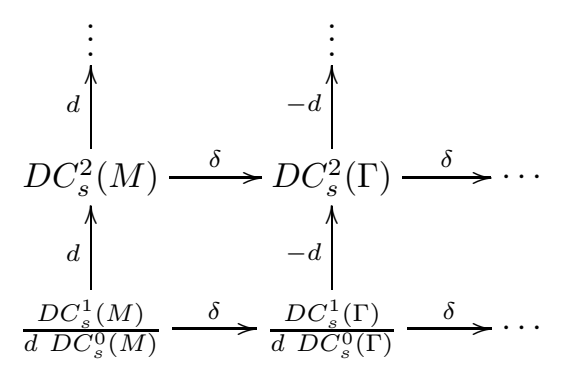

which we'll use from now on. Translating complexes into categories, we get a category $\mathcal{D C}_{s}^{2}(M \leftleftarrows \Gamma)$ associated to the second total cohomology of the above (truncated) complex (2-cocycles are objects, 1-cochains modulo exact 1-cochains are morphisms). A standard stacky argument (cf. [10]) allows one to extend the prequantization functor (on manifolds) to an equivalence

$$
\text { Preq : } \mathcal{D C}_{2}^{2}(M \leftleftarrows \Gamma) \stackrel{\sim}{\rightarrow} \mathcal{D} \mathcal{B S}^{1}(M \leftleftarrows \Gamma) .
$$

Similarly $\mathcal{D C}_{1}^{2}(M \leftleftarrows \Gamma)$ is equivalent to the category $\mathcal{B S}^{1}(M \leftleftarrows \Gamma)$ of equivariant $S^{1}$-bundles. However presently we are interested in the intermediate category $d \mathcal{B S}^{1}(M \leftleftarrows \Gamma)$ of equivariant bundles with arbitrary connections. So we consider a double complex

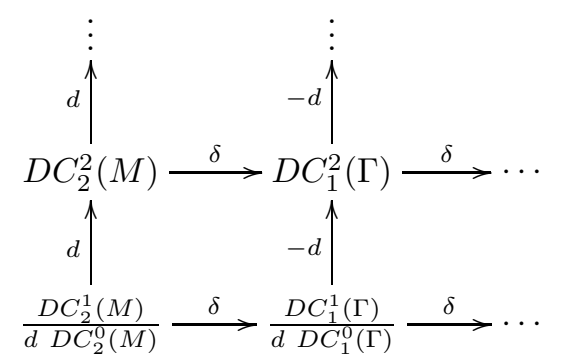

Note that truncation degree for differential forms is 2 in the first column and 1 in the second. This corresponds to the fact that equivariant structure on our bundles (i.e. isomorphism of the two pull-backs to $\Gamma$ ) does not necessarily preserve connection. Roughly speaking, we would like our characters to correspond to bundles with connections on $M$ and bundles without connections on $\Gamma$. We denote the above complex $D C_{2-1}^{2}(M \leftleftarrows \Gamma)$ and the corresponding category of total degree-2 cochains $\mathcal{D C}_{2-1}^{2}(M \leftleftarrows \Gamma)$. The complex $D C_{2-1}^{2}(M \leftleftarrows \Gamma)$ contains $D C_{2}^{2}(M \leftleftarrows \Gamma)$ as a subcomplex, and the category $\mathcal{D C}_{2-1}^{2}(M \leftleftarrows \Gamma)$ contains $\mathcal{D C}_{2}^{2}(M \leftleftarrows \Gamma)$ as a full subcategory. Explicitly, a degree- 2 cochain in $D C_{2-1}^{2}(M \leftleftarrows \Gamma$ ) (i.e an object of $\left.\mathcal{D C}_{2-1}^{2}(M \leftleftarrows \Gamma)\right)$ looks like

$$
\begin{array}{cc}
(c, h, \omega) & \bullet \\
\bullet & {[(b, f, \alpha)]}
\end{array}
$$


EQUIVARIANT DIFFERENTIAL CHARACTERS AND SYMPLECTIC REDUCTION

where $c \in C^{2}(M, \mathbb{Z}), b \in C^{1}(\Gamma, \mathbb{Z}), h \in C^{1}(M, \mathbb{R}), f \in C^{0}(\Gamma, \mathbb{R}), \omega \in \Omega^{2}(M)$, $\alpha \in \Omega^{1}(\Gamma)$, and $[(b, f, \alpha)]$ means an equivalence class with respect to the relation $(b, f, \alpha) \sim(b+d n, f-n, \alpha), n \in C^{0}(\Gamma, \mathbb{Z})$. We write this cochain as $((c, h, \omega),[(b, f, \alpha)])$. It belongs to $D C_{2}^{2}(M \leftleftarrows \Gamma)$ iff $\alpha=0$.

A degree-1 cochain in $D C_{2-1}^{2}(M \leftleftarrows \Gamma)$ (i.e a morphism in $\left.\mathcal{D C}_{2-1}^{2}(M \leftleftarrows \Gamma)\right)$ looks like

$$
[(a, t, 0)] \quad \bullet
$$

with $a \in C^{1}(M, \mathbb{Z}), t \in C^{1}(M, \mathbb{R})$, and $(a, t, 0) \sim(a+d m, t-m, 0)$ for $m \in$ $C^{0}(M, \mathbb{Z})$.

The first result of the present paper is the following prequantization theorem on groupoids:

Theorem 1.5.1. The prequantization functor

$$
\text { Preq : } \mathcal{D C}_{2}^{2}(M \leftleftarrows \Gamma) \stackrel{\sim}{\rightarrow} \mathcal{D} \mathcal{B S}^{1}(M \leftleftarrows \Gamma)
$$

extends to an equivalence of categories

$$
\text { Preq : } \mathcal{D C}_{2-1}^{2}(M \leftleftarrows \Gamma) \stackrel{\sim}{\rightarrow} d \mathcal{B S} \mathcal{S}^{1}(M \leftleftarrows \Gamma)
$$

such that if

$$
\operatorname{Preq}((c, h, \omega),[(b, f, \alpha])=(P \stackrel{\pi}{\rightarrow} M, A)
$$

then $\omega$ is the curvature of the connection $A$, while $\alpha$ is the difference of the two pull-backs of $A$ to $P_{1}$ considered as a 1-form on $\Gamma$ :

$$
\pi^{*} \alpha=\partial_{1}^{*} A-\partial_{0}^{*} A .
$$

In particular the second total cohomology group of the complex $D C_{2-1}^{\bullet}(M \leftleftarrows \Gamma)$ classifies equivariant principal $S^{1}$-bundles on $M \leftleftarrows \Gamma$ with arbitrary connections.

Proof. We give an explicit construction of the quasi-inverse functor

$$
\mathrm{DCh}: d \mathcal{B S}^{1}(M \leftleftarrows \Gamma) \rightarrow \mathcal{D C}_{2-1}^{2}(M \leftleftarrows \Gamma)
$$

of the extended prequantization functor Preq. Let us begin with objects. Given an equivariant bundle $P \rightarrow M$ with a connection $A \in \Omega^{1}(P)$ we apply DCh-functor on $M$ (cf. Subsection 1.1.1) to get a cochain $(c, h, \omega)=\operatorname{DCh}((P, A)) \in D C_{2}^{2}(M)$ such that

$$
d(c, h, \omega)=0 .
$$

Now the equivariant structure on $P$ is a bundle isomorphism $\partial_{1}^{*} P \stackrel{\sim}{\rightarrow} \partial_{0}^{*} P$ or a trivialization $\sigma: \Gamma \times S^{1} \stackrel{\sim}{\rightarrow} \partial_{1}^{*} P \otimes\left(\partial_{0}^{*} P\right)^{-1}$. We put $\sigma^{*}\left(\partial_{1}^{*} A-\partial_{0}^{*} A\right)=\alpha+d \theta$ where $\alpha \in \Omega^{1}(\Gamma)$. Applying DCh-functor on $\Gamma$ to the isomorphism $\sigma$ and using the explicit form of DCh for trivial bundles with connections (cf. Subsection 1.1) we get (a class of) cochain $\operatorname{DCh}(\sigma)=[(b, f, 0)] \in D C_{2}^{1}(\Gamma) / D C_{2}^{1}(\Gamma)$ such that

$$
d(b, f, 0)=\left(\partial_{1}^{*}(c, h, \omega)-\partial_{0}^{*}(c, h, \omega)\right)-(0, \alpha, d \alpha) .
$$

Note that $[(b, f, 0)]$ encodes not only the trivialization $\sigma$ but also natural transformations coming from the fact that the functor DCh commutes only weakly with pull-backs and products. In any case, (1.5.2) means that

$$
d(b, f, \alpha)=\delta(c, h, \omega) .
$$


Since $\sigma$ satisfies the cocycle condition on $\Gamma_{2}$ and DCh commutes strongly with pull-backs on the level of morphisms, we get

$$
\delta[(b, f, \alpha)]=0 .
$$

Equations (1.5.1), (1.5.3), (1.5.4), mean that $((c, h, \omega),[(b, f, \alpha)])$ is a cocycle in $\mathcal{D C} \mathcal{C}_{2-1}^{2}(M \leftleftarrows \Gamma)$ and we put

$$
\operatorname{DCh}((P, A, \sigma))=((c, h, \omega),[(b, f, \alpha)])
$$

This completes construction of DCh on objects of $d \mathcal{B S}^{1}(M \leftleftarrows \Gamma)$. Note that the differential form parts $\omega$ and $\alpha$ of the cocycle $((c, h, \omega),[(b, f, \alpha))])$ correspond precisely to their description in the theorem. In particular $A$ is basic iff $\alpha=0$. Hence the restriction of DCh to the subcategory $\mathcal{D B S}^{1} \subset d \mathcal{B S}^{1}$ takes values in $\mathcal{D C}_{2}^{2} \subset d \mathcal{D C} \mathcal{C}_{2-1}^{2}$ and coincides with the equivariant DCh of [10].

Since morphisms in $\mathcal{D C}_{2-1}^{2}(M \leftleftarrows \Gamma)$ (resp. $\left.d \mathcal{B S}^{1}(M \leftleftarrows \Gamma)\right)$ are the same as morphisms in $\mathcal{D C}_{2}^{2}(M)$ (resp. $\mathcal{D} \mathcal{B S}^{1}(M)$ ) we just use the original functor DCh on $M$ for morphisms.

The above construction is clearly reversible (using Preq-functors on $M$ and $\Gamma$ as quasi-inverses of DCh-functors). Hence the functor

$$
\mathrm{DCh}: d \mathcal{B S}^{1}(M \leftleftarrows \Gamma) \rightarrow \mathcal{D C}_{2-1}^{2}(M \leftleftarrows \Gamma)
$$

is an equivalence of categories and we denote the quasi-inverse functor Preq. Note that morphisms in the categories $d \mathcal{B S} \mathcal{S}^{1}(M \leftleftarrows \Gamma)$ and $\mathcal{D C}_{2-1}^{2}(M \leftleftarrows \Gamma)$ change neither the connection $A$ nor differential form parts $\omega$ and $\alpha$ of the cocycle. Therefore the condition of the theorem is satisfied for any choice of the quasi-inverse functor.

1.6. Equivariant Kostant Theorem. Recall that Kostant's prequantization theorem on a manifold $M$ states that, given a closed 2-form $\omega \in \Omega^{2}(M)$ with integral periods, there exists a principal $S^{1}$-bundle on $M$ with a connection whose curvature is equal to $\omega$. Moreover the set of such bundles with connections is in bijection with the cohomolgy $H^{1}\left(C^{\bullet}(M, \mathbb{R} / \mathbb{Z})\right)$ of the complex of smooth singular cochains with values in $\mathbb{R} / \mathbb{Z}$.

In this subsection we prove an equivariant version of Kostant theorem on a Lie groupoid $M \leftleftarrows \Gamma$. Denote $\Omega_{0}^{2}(M \leftleftarrows \Gamma) \subset \Omega^{2}(M \leftleftarrows \Gamma)=\Omega^{2}(M) \times \Omega^{1}(\Gamma) \times \Omega^{0}\left(\Gamma_{2}\right)$ the group of closed 2 -forms on $M \leftleftarrows \Gamma$ which are integral (i.e. have integral periods or, equivalently, represent images of integral cohomology classes in de Rham cohomology) and have vanishing $\Omega^{0}\left(\Gamma_{2}\right)$-component. Suppose $\Theta=(\omega, \alpha, 0) \in \Omega_{0}^{2} \subset$ $\Omega^{2}(M) \times \Omega^{1}(\Gamma) \times \Omega^{0}\left(\Gamma_{2}\right)$. Since $\Theta$ represents the image of an integral cohomology class in real smooth singular cohomology there exists an integral cochain $(c, b, a) \in$ $C^{2}(M \leftleftarrows \Gamma, \mathbb{Z})=C^{2}(M, \mathbb{Z}) \times C^{1}(\Gamma, \mathbb{Z}) \times C^{0}\left(\Gamma_{2}, \mathbb{Z}\right)$ and a real cochain $(h, f) \in$ $C^{1}(M \leftleftarrows \Gamma, \mathbb{R})=C^{1}(M, \mathbb{R}) \times C^{0}(\Gamma, \mathbb{R})$ such that $d_{\text {tot }}(h, f)=(\omega, \alpha, 0)-(c, b, a)$, where $d_{\text {tot }}$ is the total differential in the double complex of smooth singular chains on $M \leftleftarrows \Gamma$. This implies that the projection map

$$
\begin{gathered}
\eta: H^{2}\left(D C_{2-1}^{\bullet}(M \leftleftarrows \Gamma)\right) \rightarrow \Omega_{0}^{2}(M \leftleftarrows \Gamma) \\
\eta([((c, h, \omega),(b, f, \alpha),(a, 0,0))])=(\omega, \alpha, 0)
\end{gathered}
$$

is surjective, and it is easy to see that the kernel of $\eta$ is isomorphic (by the map $[((c, h, 0),(b, f, 0),(a, 0,0))] \mapsto[(h \bmod \mathbb{Z}, f \bmod \mathbb{Z})]$ to the cohomology group $H^{1}\left(C^{\bullet}(M \leftleftarrows \Gamma, \mathbb{R} / \mathbb{Z})\right)$ of smooth singular 1 -cochains with values in $\mathbb{R} / \mathbb{Z}$. Hence 
we obtain a short exact sequence

(1.6.1) $0 \rightarrow H^{1}\left(C^{\bullet}(M \leftleftarrows \Gamma, \mathbb{R} / \mathbb{Z})\right) \rightarrow H^{2}\left(D C_{2-1}^{\bullet}(M \leftleftarrows \Gamma)\right) \rightarrow \Omega_{0}^{2}(M \leftleftarrows \Gamma) \rightarrow 0$

This sequence (in the manifold case) first appeared in the original Cheeger and Simons paper [5. Since $H^{2}\left(D C_{2-1}^{\bullet}(M \leftleftarrows \Gamma)\right)$ classifies equivariant principal $S^{1}$ bundles on $M \leftleftarrows \Gamma$ with arbitrary connections (cf. Theorem[1.5.1), we can interpret (1.6.1) as follows:

Theorem 1.6.1. Let $\Theta=(\omega, \alpha, 0) \in \Omega^{2}(M \leftleftarrows \Gamma)=\Omega^{2}(M) \times \Omega^{1}(\Gamma) \times \Omega^{0}\left(\Gamma_{2}\right)$ be an integral closed 2-form on a Lie groupoid $M \leftleftarrows \Gamma$. Then there exists a $\Gamma$ equivariant principal $S^{1}$-bundle $\pi: P \rightarrow M$ with a connection $A \in \Omega^{1}(P)$ such that $\omega$ is the curvature of the connection $A$ and $\pi^{*} \alpha=\partial_{1}^{*} A-\partial_{0}^{*} A$. Moreover the set of isomorphism classes of such pairs $(P, A)$ is in bijection with $H^{1}\left(C^{\bullet}(M \leftleftarrows \Gamma, \mathbb{R} / \mathbb{Z})\right)$.

For proper groupoids this theorem was proved (by a different argument) in [2]

\section{INVARIANT CONNECTIONS AND THE MOMENT MAP}

In this section we identify differential characters corresponding to invariant connections.

2.1. The moment map. We again think of an equivariant principal $S^{1}$-bundle on a groupoid $M \leftleftarrows \Gamma$ as a simplicial bundle

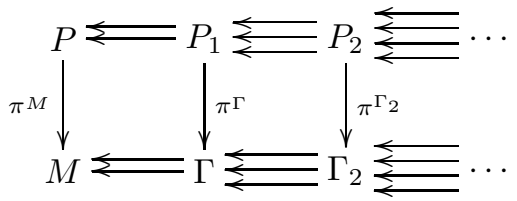

Recall (see e.g. 14) that the Lie algebroid $\mathfrak{P}$ of the Lie groupoid $P \leftleftarrows P_{1}$ is a vector bundle over $P$ whose fiber at $p \in P$ is equal to $\operatorname{Ker} d s \subset T_{p} P_{1}$ (here we consider $P$ as a subset of $P_{1}$ via the unit map). Another way to think of sections of $\mathfrak{P}$ is as right-invariant vector fields in Ker $d s \subset T P_{1}$. The Lie algebroid $\mathfrak{P}$ comes equipped with a bundle map $\rho:=d t: \mathfrak{P} \rightarrow T P$ called the anchor.

Let $\mathfrak{P}^{*}$ be the dual bundle of $\mathfrak{P}$. Given a connection $A \in \Omega^{1}(P)$ we define the moment map $\mu_{A}^{P}$ as a section of $\mathfrak{P}^{*}$ given by:

$$
\mu_{A}^{P}(X)=A(\rho(X))
$$

for a section $X$ of $\mathfrak{P}$. One can rewrite this definition as

$$
\begin{aligned}
\mu_{A}^{P}(X)=A(d t(X))=A(d t(X)- & d s(X))= \\
& =\left(d t^{*}(A)-d s^{*}(A)\right)(X)=(\delta A)(X) .
\end{aligned}
$$

Here $\delta$ is, as usual, the simplicial differential, and we think of $X$ as a right-invariant section of Ker $d s \subset T P_{1}$ and of $\mu_{A}^{P}(X)$ as a right-invariant function on $P_{1}$, hence a function on $P$.

Since a connection on and all the structure maps of an simplicial principal $S^{1}$ bundle are preserved under the action of $S^{1}$, the moment map $\mu_{A}^{P}$ is a pullback under $\pi^{M}$ of a section $\mu_{A}^{M}$ of the dual bundle $\mathfrak{G}^{*}$ of the Lie algebroid $\mathfrak{G}$ of the base groupoid $M \leftleftarrows \Gamma$. Without much risk of confusion we call both $\mu_{A}^{M}$ and $\mu_{A}^{P}$ the moment maps and often drop the top index. By definition a connection $A$ is horizontal iff $\mu_{A}=0$. 
For example, the Lie algebroid of an action groupoid $P \leftleftarrows G \times P$ is the trivial bundle $P \times \mathfrak{g}$ and the moment map is a map $\mu: P \rightarrow \mathfrak{g}^{*}$ given by $\langle\mu, X\rangle=i_{\rho(X)} A$, $X \in \mathfrak{g}$.

2.2. Invariant connections. We continue with the setup of the previous subsection. Our goal is to define what it means for a connection to be $P_{1}$-invariant. In the case of a global action $P \leftleftarrows G \times P$ a connection $A \in \Omega^{1}(P)$ is $G$-invariant iff $g^{*} A=A$ for any $g \in G$. In the groupoid language an element $g \in G$ should be thought of as a constant section $\Sigma$ of the source map $s$, and the condition $g^{*} A=A$ can be written as

$$
\left.\left(t^{*} A-s^{*} A\right)\right|_{T \Sigma}=0
$$

or

$$
\left.(\delta A)\right|_{T \Sigma}=0
$$

For a general (non-action) groupoid there is no natural choice of the section $\Sigma$ through a point $p \in P_{1}$. One can fix a local regular foliation $\Sigma$ of $P_{1}$ by bisections (submanifolds transverse to both $s$ and $t$ ) and say that $A$ is $\Sigma$-invariant if (2.2.1) holds. This condition depends on the choice of $\Sigma$.

Note that we have a decomposition

$$
T P_{1}=T \Sigma \oplus \operatorname{Ker} d s
$$

Hence the two equations (2.2.1) and (2.1.1) completely determine $\delta A$. In particular, if the moment map $\mu$ vanishes at some point $p \in P_{1}$ then the condition (2.2.1) at the point $p$ does not depend on the choice of $\Sigma$. If $\mu$ vanishes identically than the choice of $\Sigma$ is irrelevant and the connection is invariant (for some $\Sigma$ ) iff it is basic $(\delta A=0)$. In other words, as in the global quotient case, the connection is basic iff it is horizontal and invariant. However for a general groupoid the second condition is natural only if the first one is satisfied.

2.3. Prequantization on global quotients. We would like to characterize bundles with invariant connections via their differential characters. Let us restrict ourselves to a global quotient case $M \leftleftarrows G \times M$ to avoid choice of a local foliation $\Sigma$. Given an equivariant bundle $P$ with an invariant connection $A$ we apply the functor DCh (cf. Theorem 1.5.1) to obtain a character

$$
\operatorname{DCh}((P, A))=((c, h, \omega),[(b, f, \alpha)])
$$

where $\pi^{*} \alpha=\delta A \in \Omega^{1}(G \times P)$. Then, according to the previous subsection, $G$ invariance of the connection $A$ is equivalent to the following vanishing condition on the form $\alpha \in \Omega^{1}(G \times M)$ :

$$
i_{v} \alpha=0
$$

for any vector field $v \in T M \subset T(G \times M)=T G \oplus T M$. This means that

$$
\alpha=<\mu, d g g^{-1}>\text {, }
$$

where $d g g^{-1}$ is the right-invariant Maurer-Cartan form on $G$ (pulled back to $G \times M$ ) and $\mu: M \rightarrow \mathfrak{g}^{*}$ is the moment map (pulled back to $\left.G \times M\right)$.

Putting everything together we obtain the following description of equivariant bundles with invariant connections. 
Theorem 2.3.1. Suppose a Lie group $G$ acts on a manifold $M$. Then the prequantization functor

$$
\text { Preq : } \mathcal{D C}_{2-1}^{2}(M \leftleftarrows G \times M) \stackrel{\sim}{\rightarrow} d \mathcal{B S}^{1}(M \leftleftarrows G \times M)
$$

restricts to an equivalence

$$
\text { Preq : } \mathcal{D C}_{\text {inv }}^{2}(M \leftleftarrows G \times M) \stackrel{\sim}{\rightarrow} d \mathcal{B} \mathcal{S}_{\text {inv }}^{1}(M \leftleftarrows G \times M),
$$

where $d \mathcal{B S}_{\text {inv }}^{1}(M \leftleftarrows G \times M)$ is the category of $G$-equivariant bundles with $G$ invariant connections and $\mathcal{D} \mathcal{C}_{\mathrm{inv}}^{2}(M \leftleftarrows G \times M)$ is the category of differential characters of the form $\left((c, h, \omega),\left[\left(b, f,<\mu, d g g^{-1}>\right)\right]\right)$.

To summarize we have two towers of full subcategories related by prequantization/character equivalence:

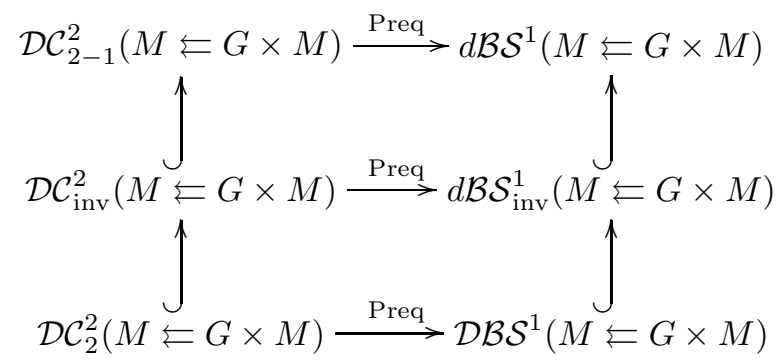

The bottom row consists of equivariant bundles with basic connections and characters with vanishing $\alpha$-component; the middle row bundles have invariant connections and the characters have $\alpha=<\mu, d g g^{-1}>$; finally the top row does not involve any conditions on the connection or $\alpha$-component of the character.

\section{REDUCTION}

The idea of reduction is that after restriction to the zero level of the moment map an invariant connection becomes basic and thus defines a connection on a bundle on the quotient stack. From our point of view it is more natural to consider categories of bundles rather than a single bundle. Hence we start with the "zero level set" and consider all bundles with the moment map vanishing on that manifold.

3.1. General Reduction. Let $M \leftleftarrows \Gamma$ be a Lie groupoid, $N$ a submanifold of $M$ such that $s^{-1} N=t^{-1} N, i: N \rightarrow M$ the inclusion map, and $N \leftleftarrows \Gamma_{N}$ the corresponding full subgroupoid of $M \leftleftarrows \Gamma$ (i.e. $\Gamma_{N}=\Gamma \times{ }_{s} M_{i} N=\Gamma \times{ }_{t} M_{i} N$ ).

We denote by $d \mathcal{B S}_{N}^{1}(M \leftleftarrows \Gamma)$ the full subcategory of $d \mathcal{B S}^{1}(M \leftleftarrows \Gamma)$ consisting of equivariant principal $S^{1}$-bundles with connections $(P, A)$ such that the restriction $i^{*}(P, A)$ of $(P, A)$ to $N$ is an equivariant bundle with a basic connection (i.e. such that $\left.i^{*} s^{*} A=i^{*} t^{*} A\right)$. As explained in the previous section, this condition can be split into two:

(3.1.i) the moment map of $A$ vanishes on $N:\left.\mu_{A}\right|_{N}=0$;

(3.1.ii) $i^{*} A$ is $\Gamma_{N}$-invariant (with respect to any bisection).

Similarly we define a full subcategory $\mathcal{D C}_{N}^{2}(M \leftleftarrows \Gamma)$ of $\mathcal{D C} \mathcal{C}_{2-1}^{2}(M \leftleftarrows \Gamma)$ consisting of differential characters $((c, h, \omega),[(b, f, \alpha)])$ such that $\left.\alpha\right|_{\Gamma_{N}}=0$.

It follows from Theorem 1.5.1 that the prequantization functor

$$
\text { Preq }: \mathcal{D C}_{2-1}^{2}(M \leftleftarrows \Gamma) \stackrel{\sim}{\rightarrow} d \mathcal{B S}^{1}(M \leftleftarrows \Gamma)
$$


restricts to an equivalence

$$
\text { Preq : } \mathcal{D C}_{N}^{2}(M \leftleftarrows \Gamma) \stackrel{\sim}{\rightarrow} d \mathcal{B S}_{N}^{1}(M \leftleftarrows \Gamma)
$$

The restriction $i^{*} d \mathcal{B S}_{N}^{1}(M \leftleftarrows \Gamma)$ of the category $d \mathcal{B S}_{N}^{1}(M \leftleftarrows \Gamma)$ to the submanifold $N$ is equivalent to the category $\mathcal{D B S}^{1}\left(\left.N \leftleftarrows \Gamma\right|_{N}\right)$ of equivariant bundles with basic connections on $N$, and similarly $i^{*} \mathcal{D} \mathcal{C}_{N}^{2}(M \leftleftarrows \Gamma) \simeq \mathcal{D C}_{2}^{2}\left(N \leftleftarrows \Gamma_{N}\right)$. Hence we obtain the following result:

Theorem 3.1.1. Prequantization commutes with reduction, i.e. the following diagram commutes up to natural transformations:

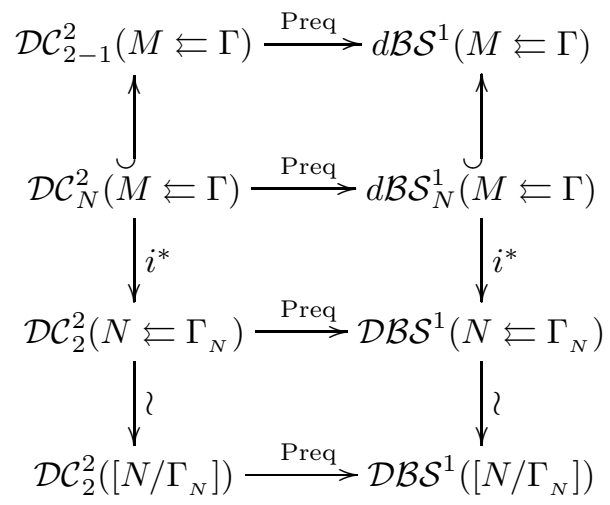

Note that prequantization functors in the above diagram are defined differently for each row (the top two rows are constructed in the present paper while the bottom two are from [10]). The meaning of the theorem is that these definitions are compatible.

3.2. Reduction for action groupoids. On an action groupoid $M \leftleftarrows G \times M$ the reduction can be performed in a more familiar way: first consider subcategories $d \mathcal{B S}_{\text {inv }}^{1}(M \leftleftarrows G \times M), \mathcal{D C}_{\text {inv }}^{2}(M \leftleftarrows G \times M)$ of invariant connection/characters and then further subcategories $d \mathcal{B S}_{\text {inv }, N}^{1}(M \leftleftarrows G \times M), \mathcal{D C}_{\text {inv }, N}^{2}(M \leftleftarrows G \times M)$ of invariant connection with the moment map vanishing on a given $G$-stable submanifold $N$. The categories $d \mathcal{B S}_{\text {inv }, N}^{1}(M \leftleftarrows G \times M), \mathcal{D C}_{\text {inv }, N}^{2}(M \leftleftarrows G \times M)$ are in general proper subcategories of $d \mathcal{B S}_{N}^{1}(M \leftleftarrows G \times M), \mathcal{D C}_{N}^{2}(M \leftleftarrows G \times M)$ introduced in the previous subsection since in the latter ones we impose invariance condition only on the submanifold $N$.

This version of reduction also commutes with prequantization. 
Theorem 3.2.1. The diagram

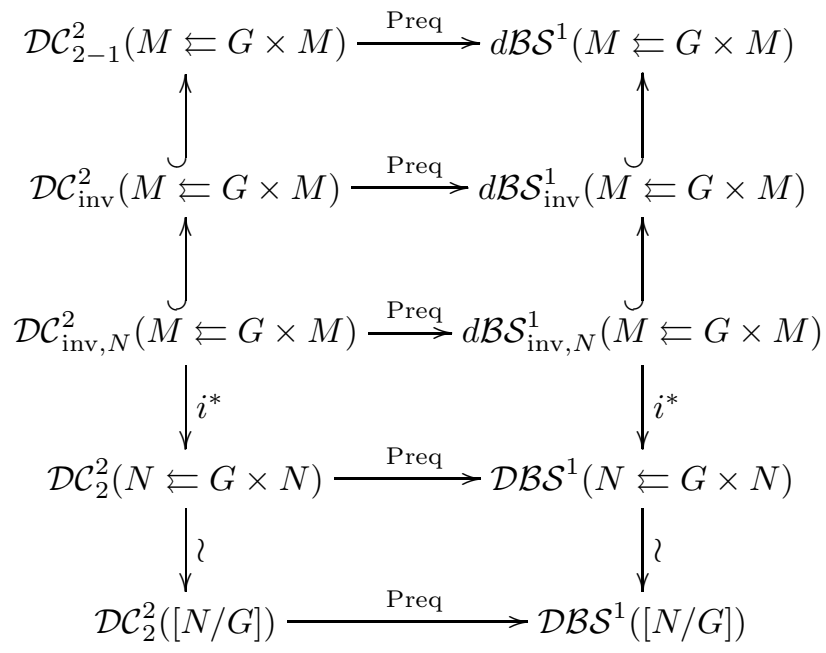

commutes up to natural transformations.

The proof is again a simple application of Theorem 1.5.1 (more precisely, of the explicit relation between $\delta A$ and the $\alpha$-part of a differential character).

\section{Differential Characters as Characters}

In this section we provide an explicit description of the actual differential characters on groupoids, i.e. of the isomorphism classes of objects of $\mathcal{D C}_{2-1}^{2}(M \leftleftarrows \Gamma)$ or, in other words, of the second total cohomology group of the complex $D C_{2-1}^{\bullet}(M \leftleftarrows \Gamma)$.

4.1. Differential characters on manifolds and groupoids. Let us first recall the description of Cheeger-Simons differential characters in the case of a manifold $M$ (cf. [5, 8]). We are interested in the cohomology group

$$
\begin{aligned}
& H^{2}\left(D C_{2}^{\bullet}(M)=\right. \\
= & \frac{\left\{(c, h, \omega) \in C^{2}(M, \mathbb{Z}) \times C^{1}(M, \mathbb{R}) \times \Omega^{2}(M) \mid d c=0, \omega-c-d h=0, d \omega=0\right\}}{(c, h, \omega) \sim(c+d a, h-a-d t, \omega),(a, t) \in C^{1}(M, \mathbb{Z}) \times C^{0}(M, \mathbb{R})}
\end{aligned}
$$

It is easy to see that equivalence (cohomology) class of $(c, h, \omega)$ is completely determined by $\omega$ and the values of $h \bmod \mathbb{Z}$ on smooth 1-cycles. To make a precise statement let us introduce the group of differential characters on $M$. A differential character is a pair $(\omega, \chi)$, where $\omega \in \Omega^{2}(M), d \omega=0$, and $\chi$ is a character $Z_{1}(M) \rightarrow \mathbb{R} / \mathbb{Z}$ of the group of smooth 1 -cycles $Z_{1}(M)$. This pair should satisfy the following condition:

$$
\chi(\partial S)=\int_{S} \omega \bmod \mathbb{Z}
$$

for any smooth 2-chain $S \in C_{2}(M, \mathbb{Z})$. The map

$$
[(c, h, \omega)] \mapsto\left(\omega,\left.h\right|_{Z_{1}(M)} \bmod \mathbb{Z}\right)
$$

provides an isomorphism between $H^{2}\left(D C_{2}^{\bullet}(M)\right)$ and the group of differential characters. Condition (4.1.1) ensures that $c=\omega-d h$ is an integral cochain. 
Let us turn to the groupoid case. We would like to have an explicit description of the second total cohomology group of the complex $D C_{2-1}^{\bullet}(M \leftleftarrows \Gamma)$. An element of $H^{2}\left(D C_{2-1}^{\bullet}(M \leftleftarrows \Gamma)\right)$ is a closed cochain $((c, h, \omega),[(b, f, \alpha)])$ up to addition of the total differential of $[(a, t, 0)]$, see 1.5 for notation. The cochain being closed means

$$
\begin{aligned}
d(c, h, \omega) & =0 \\
\delta(c, h, \omega) & =d[(b, f, \alpha)] \\
\delta[(b, f, \alpha)] & =0
\end{aligned}
$$

Similar to the manifold case $c$ and $b$ are determined by $h, f, \omega$ and $\alpha$ :

$$
\begin{aligned}
& b=\alpha-d f-\delta h \\
& c=\omega-d h
\end{aligned}
$$

Turning to differential form components, we put

$$
\Theta=(\omega, \alpha, 0) \in \Omega^{2}(M) \oplus \Omega^{1}(\Gamma) \oplus \Omega^{0}\left(\Gamma_{2}\right)=\Omega^{2}(M \leftleftarrows \Gamma) .
$$

Then $\Theta$ is a closed 2 -form on $M \leftleftarrows \Gamma$ with vanishing $\Omega^{0}\left(\Gamma_{2}\right)$-component.

Finally the pair $(h, f)$ is determined up to coboundary (total differential of $[(a, t, 0)])$ by its values $\bmod \mathbb{Z}$ on smooth 1 -cycles $Z_{1}(M \leftleftarrows \Gamma)$. Recall that a cycle (generator of $Z_{1}$ ) on a groupoid looks like this (see for example [1]):

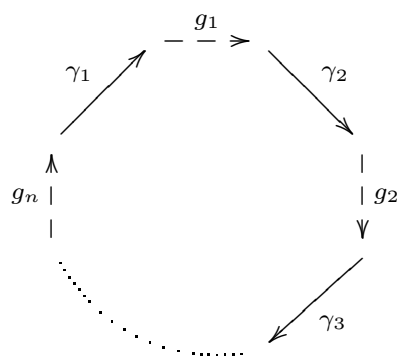

Here $\gamma_{i}:[0,1] \rightarrow M$ are smooth paths in $M$ and $g_{i} \in \Gamma$ are arrows connecting endpoints of these paths. This is a particular example of a smooth singular 1-chain on the groupoid and so it makes sense to evaluate the cochain $(h, f)$ on this chain: $\sum h\left(\gamma_{i}\right)+\sum f\left(g_{i}\right) \in \mathbb{R}$. Taking values $\bmod \mathbb{Z}$ we get a character

$$
\Psi=\left.(h, f)\right|_{Z_{1}(M \leftleftarrows \Gamma)} \bmod \mathbb{Z} \quad: \quad Z_{1}(M \leftleftarrows \Gamma) \rightarrow \mathbb{R} / \mathbb{Z} .
$$

The pair $(\Theta, \Psi)$ uniquely determine the cohomology class of $((c, h, \omega),[(b, f, \alpha)])$. Conversely, a pair $\left(\Theta \in \Omega^{2}(M \leftleftarrows \Gamma), \Psi: Z_{1}(M \leftleftarrows \Gamma) \rightarrow \mathbb{R} / \mathbb{Z}\right)$ corresponds to a cohomology class in $H^{2}\left(D C_{2-1}^{\bullet}(M \leftleftarrows \Gamma)\right)$ iff it satisfies the following conditions:

(4.1.i) $\Theta$ is a closed 2 -form on $(M \leftleftarrows \Gamma)$ with vanishing $\Omega^{0}\left(\Gamma_{2}\right)$-component. This ensures that the third components of the relations (4.1.2) - (4.1.4) hold true.

(4.1.ii) $\Psi\left(g_{1} g_{2} g_{3}\right)=0$ for $g_{1}, g_{2}, g_{3} \in \Gamma$ such that $g_{1} g_{2} g_{3}=i d_{p}, p \in M$. Note that such a triple $g_{1}, g_{2}, g_{3}$ is a cycle in $Z_{1}(M \leftleftarrows \Gamma)$ :

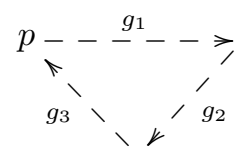


This condition ensures that $\delta f=0$.

(4.1.iii) For any smooth path $\eta:[0,1] \rightarrow \Gamma$ one has

$$
\Psi(\partial \eta)=\int_{\eta} \alpha \bmod \mathbb{Z}
$$

where $\partial \eta$ is the total boundary of $\eta$, i.e. the following cycle in $Z_{1}(M \leftleftarrows \Gamma)$ :

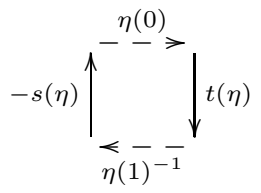

This condition ensures that $b(\eta)$ defined by (4.1.5) belongs to $\mathbb{Z}$.

(4.1.iv) For any smooth 2-chain $S$ on $M$ one has

$$
\Psi(\partial S)=\int_{S} \omega \bmod \mathbb{Z} .
$$

Note that $\partial S$ is a 1-cycle on $M$ but we think of it as an element of $Z_{1}(M \leftleftarrows \Gamma)$

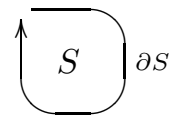

This condition ensures that $c(S)$ defined by (4.1.6) belongs to $\mathbb{Z}$.

Let us summarize the above discussion as the following version of Theorem 1.5.1,

Theorem 4.1.1. Isomorphism classes of equivariant $S^{1}$-bundles with (not necessarily basic) connections on a groupoid $M \leftleftarrows \Gamma$ are in bijection with differential characters, i.e. pairs $(\Theta, \Psi)$, where $\Theta \in \Omega^{2}(M \leftleftarrows \Gamma)$ and $\Psi$ is a character $Z_{1}(M \leftleftarrows \Gamma) \rightarrow \mathbb{R} / \mathbb{Z}$, satisfying conditions 4.1.i) (4.1.iv above.

Characters $(\Theta, \Psi)$ with vanishing $\Omega^{1}(\Gamma)$-component of $\Theta$ correspond to equivariant bundles with basic connections, i.e. bundles with connections on the quotient stack.

Remark. Note that $\left(\left.\Theta\right|_{M},\left.\Psi\right|_{Z_{1}(M)}\right)$ defines a differential character on $M$ corresponding to an $S^{1}$-bundle with connection on $M$. The above Theorem says that equivariant structures on this bundle are in 1-1 correspondence with equivariant extensions of its character.

4.2. Special cases. Because of conditions (4.1.ii) - (4.1.iv) the character $\Psi$ is determined by its value on the following classes of 1-cycles for each point $p \in M$ : (1) generators of the fundamental group of $M$ with the base point $p,(2)$ representatives of the connected components of the inertia group $I_{p}=s^{-1} p \cap t^{-1} p$, and (3) cycles of the form

$$
p \underset{-\ldots}{-},
$$

where as before the solid line is a path in $M$ and the broken line is an arrow in $\Gamma$. Moreover it is enough to consider one base point $p$ in each connected (via sequences of paths in $M$ and arrows in $\Gamma$ ) component of $M$. This description of $\Psi$, though economical, is not very natural because homotopy of paths/points involves integration of $\omega$ and/or $\alpha$. However in some cases it can clarify (and simplify) things substantially. Here are some examples: 
(4.2.i) If $\Theta=0$ then $\Psi$ is just a homomorphism of the product of the (suitably defined) fundamental groups of connected components of the stack $[M / \Gamma]$ into $\mathbb{R} / \mathbb{Z}$, i.e. a local system on $[M / \Gamma]$.

(4.2.ii) If the groupoid is source-connected (e.g. it is the action groupoid of a connected group action) then $\Psi$ is determined by its values on $Z_{1}(M)$ (or just on generators of fundamental groups of connected components of $M)$.

(4.2.iii) For any point $p \in M$ the character $\Psi$ restricts to a homomorphism $I_{p} \rightarrow \mathbb{R} / \mathbb{Z}$, where $I_{p}$ is the inertia group at $p$. Moreover this homomorphism is determined by its values on representatives of the connected components of $I_{p}$. Note however that, unless the quotient of $I_{p}$ by its identity component $I_{p}^{e}$ has a section or the form $\alpha$ vanishes, $\Psi$ does not lift to a homomorphism $I_{p} / I_{p}^{e} \rightarrow \mathbb{R} / \mathbb{Z}$.

(4.2.iv) In the case of a finite group $G$ acting on a point, a differential character is just a homomorphism $G \rightarrow \mathbb{R} / \mathbb{Z}$.

4.3. Invariant differential characters on an action groupoid. In the case of an action groupoid $M \leftleftarrows G \times M$ we have a notion of invariant connections (cf. subsection 2.3) which fit between arbitrary and basic ones. We would like to describe the corresponding differential characters. Invariance of a connection corresponds to vanishing of the form $\alpha \in \Omega^{1}(G \times M)$ on constant sections of the source map (projection) $s: G \times M \rightarrow M$. Because of relation 4.1.iii) this vanishing condition is equivalent to vanishing of the character $\Psi$ on cycles of the type

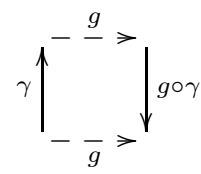

Here $\gamma:[0,1] \rightarrow M$ is a path in $M$, and we denote by the same letter $g$ an element of $G$, the corresponding constant section of $s$, and the corresponding diffeomorphism of $M$. Taking into account (4.1.iil) we see that invariance of the connection means that the character $\Psi$ is invariant with respect to the natural action of $g \in G$ on $Z_{1}(M \leftleftarrows M \times G)$.

Note that assuming (4.1.i), (4.1.ii), and (4.1.iv), it is enough to require (4.1.iii) on homotopy representatives of paths in $G \times M$ and any path in $G \times M$ is homotopic to a composition of a constant section of $s$ and a 1-parametric subgroup in $G$ acting on a point in $M$. The above invariance condition takes care of constant sections of $s$, so let us consider a 1-parametric subgroup $e^{t X}, X \in \mathfrak{g}$. Given a point $p \in M$ the relation (4.1.iii) applied to the path $\left(e^{t X}, p\right) \subset G \times M$ reads

$$
\Psi\left(p \underset{e_{-}^{-X}-}{e^{t X}} e^{X} p\right)=<\mu(p), X>\bmod \mathbb{Z} .
$$

Here the solid arrow represents the path $e^{t X} p, t \in[0,1]$, in the manifold $M$, the broken arrow the element $\left(e^{t X}, p\right)$ of $G \times M$, and $\mu: M \rightarrow \mathfrak{g}^{*}$ is the moment map.

This discussion together with subsection 2.3. leads us to the following description of the invariant characters for an action groupoid. 
Theorem 4.3.1. Isomorphism classes of equivariant $S^{1}$-budles with invariant connections on an action groupoid $M \leftleftarrows G \times M$ are in bijection with invariant differential characters, i.e. triples $(\omega, \mu, \Psi)$, where $\omega \in \Omega^{2}(M)$ is a 2-form, $\mu: M \rightarrow \mathfrak{g}^{*}$ is a (moment) map, and $\Psi$ is a character $Z_{1}(M \leftleftarrows G \times M) \rightarrow \mathbb{R} / \mathbb{Z}$. This triple should satisfy the following conditions:

(4.3.i) $\omega$ and $\Psi$ are $G$-invariant, $\mu$ is $G$-equivariant.

(4.3.ii) $i_{\varepsilon_{X}} \omega=-<d \mu, X>$, where $X \in \mathfrak{g}$ and $\varepsilon_{X}$ is the action vector field of $X$.

(4.3.iii) $\left(\omega,\left.\Psi\right|_{Z_{1}(M)}\right)$ is an (invariant) differential character on $M$, i.e.

$$
\Psi(\partial S)=\int_{S} \omega \bmod \mathbb{Z}
$$

(4.3.iv) One has

$$
\Psi\left(p \underset{e_{e^{-X}}-}{e^{t X}} e^{X} p\right)=<\mu(p), X>\bmod \mathbb{Z}
$$

for any $p \in M, X \in \mathfrak{g}$.

(4.3.v) $\Psi$ restricts to a character $\Psi_{p}: I_{p} \rightarrow \mathbb{R} / \mathbb{Z}$ of the stabilizer (inertia) group $I_{p}$ for each point $p \in M$.

The connection is basic iff $\mu \equiv 0$.

Here we rewrote the condition that the form $\left(\omega,<\mu, d g g^{-1}>, 0\right) \in \Omega^{2}(M \leftleftarrows G \times M)$ is closed in a more familiar form (see for example [7]): (1) $\omega$ is closed and $G$ invariant, (2) $\mu: M \rightarrow \mathfrak{g}^{*}$ is $G$-equivariant, and (3) $i_{\varepsilon_{X}} \omega=-\langle d \mu, X>$.

As for a general groupoid, one only needs to specify the character $\Psi$ on some elements of $Z_{1}(M \leftleftarrows G \times M)$. However a natural choice of these generators depends on the particular situation.

\section{EXAMPLES}

In this section we sketch several examples of differential characters and reduction. We leave the details to an interested reader.

5.1. Example: classifying stack. Consider a Lie group $G$ acting on a point $*$. A differential character on the groupoid $* \leftleftarrows G \times *$ is a homomorphism $\Psi: G \rightarrow$ $\mathbb{R} / \mathbb{Z}$ together with an element $\mu \in \mathfrak{g}^{*}$, such that $d \Psi=\mu$. Such a pair $(\Psi, \mu)$ is determined by the character $\Psi$ which has to be smooth. So the differential character on $* \leftleftarrows G \times *$ is the same as a smooth character of $G$.

5.2. Example: coadjoint orbits of compact Lie groups. Let $M$ be a coadjoint orbit of a compact connected Lie group $G: M=G \cdot \lambda$ for $\lambda \in \mathfrak{g}^{*}$. If we assume the moment map $\mu: M \hookrightarrow \mathfrak{g}^{*}$ the inclusion then the rest of a differential character is uniquely determined by Theorem 4.3.1 as follows.

Let $\tau: G \rightarrow M$ be given by $\tau(g)=g \cdot \lambda$. The condition (4.3.iil) implies

$$
\tau^{*} \omega=d<\lambda, g^{-1} d g>,
$$

where $g^{-1} d g$ is the left-invariant Maurer-Cartan form. This equality completely determines $\omega$ since $\tau$ is a surjective submersion. 
Now we turn to the character $\Psi$. Note that $\Psi$ is $G$-invariant and $G$ is compact. Therefore $\Psi$ is determined by its values on $G$-invariant cycles in $Z_{1}(G \times M)$ containing $\lambda$. Moreover, $G$ being connected, we can assume these cycles to be closed paths

$$
e^{t X} \cdot \lambda \subset M, \quad 0 \leq t \leq 1, \quad X \in \mathfrak{g},
$$

produced by actions of 1-parametric subgroups of $G$ on $\lambda$. For such cycles equation 4.3.iv implies

$$
\Psi\left(\left\{e^{t X} \cdot \lambda\right\}\right)=<\lambda, X>+\Psi\left(e^{X}\right) \bmod \mathbb{Z}
$$

Note that $e^{X}$ belongs to the stabilizer $I_{\lambda}$ of $\lambda$ in $G$ (in other words to the inertia group at $\lambda$ ); we denote $\mathfrak{i}_{\lambda}$ the Lie algebra of $I_{\lambda}$. It remains to determine the values of the character $\left.\Psi\right|_{I_{\lambda}}: I_{\lambda} \rightarrow \mathbb{R} / \mathbb{Z}$. Because of (4.3.iv) we have $\left.\mathrm{d} \Psi\right|_{I_{\lambda}}=\left.\lambda\right|_{\mathfrak{i}_{\lambda}}$ and $I_{\lambda}$ being connected this determines $\left.\Psi\right|_{I_{\lambda}}$, completing construction of the differential character $(\omega, \mu, \Psi)$.

Of course there are still conditions to be checked for $(\omega, \mu, \Psi)$ to be a differential character. For example, $\left.\lambda\right|_{i_{\lambda}}$ should actually lift to a character of the stabilizer group $I_{\lambda}$. In fact (we don't prove it in the present paper), this is the only condition (for existence of a differential character on the coadjoint orbit of $\lambda \in \mathfrak{g}^{*}$ ). One can say that differential character on an orbit is induced from a smooth character of the stabilizer group of a point on the orbit.

Let us remark that usually the integrality condition is stated in terms of the form $\omega$ on $M$ and then additional conditions on $\lambda$ are imposed to ensure that the Lie algebra action on the prequantization bundle lifts to an action of the group. For example, if $G$ is simply connected then the differential character exists iff $\omega$ is integral. Our point of view is that it is more natural to emphasise the character $\Psi$ rather than the form $\omega$. As an extreme example, if $G=T$ is a torus (compact connected abelian group) then each coadjoint orbit is a point $\lambda \in \mathfrak{t}^{*}$, hence has unique (integral) 2 -form $\omega(=0)$. However only those points for which $\lambda$ is a differential of a character of $T$ are prequantizable according to the above discussion. In fact the set of prequantizable orbits in $t^{*}$ is the lattice of characters of $T$. Moreover, since an orbit is a point, the differential character is just a smooth character of $T$.

5.3. Example: coadjoint orbits of $S U(2)$ and $S^{1}$-reduction. Let $S^{2}$ be a sphere about the origin in $\mathbb{R}^{3}$. We think of $S^{2}$ as a coadjoint orbit of $S U(2)$ in $\mathfrak{s u} * \approx \mathbb{R}^{3}$. In particular, $S U(2)$ acts on $S^{2}$ by rotations (via the 2 -fold covering $\operatorname{map} S U(2) \rightarrow S O(3, \mathbb{R}))$.

It is easy to see that given an integral 2-form $\omega \in \Omega^{2}\left(S^{2}\right)$ one has unique $S U(2)$ invariant differential character $(\omega, \mu, \Psi)$ on $S^{2}$. For example, let us compute the restriction $\chi:\{ \pm 1\} \rightarrow \mathbb{R} / \mathbb{Z}$ of the character $\Psi$ to the center $\{ \pm 1\} \subset S U(2)$. Here we think of the center as a subgroup of the inertia group $I_{p}$ at a point $p \in S^{2}$ (note that $\Psi$ is $S U(2)$-invariant and hence $\chi$ does not depend on the point $p$ ). Let $X$ be (unique up to sign) element of the Lie algebra $\mathfrak{s u}_{2}$ such that $e^{X}=-1 \in S U(2)$, $<\mu(p), X>=0$, and $\gamma(t)=e^{t X} p, 0<t<1$, is a big circle on $S^{2}$ bounding a half-sphere $B \subset S^{2}$. Then we have

$$
\chi(-1)=\Psi\left(e^{X}\right) \stackrel{\text { 4.3.iv }}{=} \Psi(\partial B) \stackrel{\text { 4.3.iii) }}{=} \int_{B} \omega=\frac{1}{2} \int_{S^{2}} \omega \bmod \mathbb{Z} .
$$


In fact, we did not have to assume that $\left\langle\mu(p), X>=0\right.$. For an arbitrary $X \in \mathfrak{s u}_{2}$ such that $e^{X}=-1$, one has

$$
\begin{aligned}
\chi(-1)=\Psi\left(e^{X}\right) \stackrel{\text { 4.3.iv }}{=} \Psi(\partial B)-<\mu(p), X>\stackrel{4.3 \text {.iii }}{=} \int_{B} \omega-<\mu(p), X>= \\
=\frac{1}{2} \int_{S^{2}} \omega \bmod \mathbb{Z},
\end{aligned}
$$

where $B$ is either of the two parts of $S^{2}$ bounded by the circle $\gamma(t)=e^{t X} p, 0<t<1$, and the last equality follows from Archimedes (or Duistermaat-Heckman) Theorem.

Let us now turn to the reduction procedure with respect to the action of a torus $T \approx S^{1} \subset S U(2)$. We denote by $h$ the restriction of the moment map $\mu$ to the Lie algebra of $T$ (so if $T$ is the group of rotations about the vertical axis, then $h$ is the height function on $S^{2}$ up to scale). According to Section 3 the reduction is the following sequence of functors (cf. (3.2.1)):

$$
\begin{aligned}
\mathcal{D} \mathcal{C}_{\text {inv }}^{2}\left(S^{2} \leftleftarrows S U(2) \times\right. & \left.S^{2}\right) \rightarrow \mathcal{D C}_{\text {inv }}^{2}\left(S^{2} \leftleftarrows T \times S^{2}\right) \rightarrow \\
& \rightarrow \mathcal{D C}_{2}^{2}\left(h^{-1}(0) \leftleftarrows T \times h^{-1}(0)\right) \stackrel{\sim}{\rightarrow} \mathcal{D} \mathcal{C}_{2}^{2}(p \leftleftarrows\{ \pm 1\} \times p) .
\end{aligned}
$$

Here $p$ is a point on the circle $h^{-1}(0)$, the first arrow is the restriction, the second is the actual reduction (i.e. restriction to the zero level of the moment map), and the last (restriction) isomorphism is due to the fact that $\mathcal{D C} \mathcal{C}_{2}^{2}$ is a stack.

The category $\mathcal{D C}_{2}^{2}(p \leftleftarrows\{ \pm 1\} \times p)$ is just the category of characters (or 1-dim representations) of the group $\{ \pm 1\}$. On the other hand, an isomorphism class of an object $(c, h, \omega)$ of $\mathcal{D} \mathcal{C}_{\text {inv }}^{2}\left(S^{2} \leftleftarrows T \times S^{2}\right)$ is determined by $\omega$. Since the reduction is just the restriction of differential characters we see that it takes $\omega$ to the character $\chi:\{ \pm 1\} \rightarrow \mathbb{R} / \mathbb{Z}$ given, as above, by

$$
\chi(-1)=\frac{1}{2} \int_{S^{2}} \omega \bmod \mathbb{Z} .
$$

One of our main motivations for developing the theory of invariant differential characters was to provide a context for the above formula. It corresponds to the fact that an irreducible representation of $S U(2)$ has a $T$-invariant vector iff its highest weight is even.

\section{REFERENCES}

[1] K. Behrend, Cohomology of stacks, Intersection theory and moduli, ICTP Lect. Notes, XIX, 2004, pp. 249-294 (electronic). MR 2172499

[2] K. Behrend and P. Xu, Differentiable Stacks and Gerbes, available at arXiv:math.DG/0605694.

[3] A. Bellinson, Higher regulators and values of L-functions, Current problems in mathematics, Vol. 24, 1984, pp. 181-238 (Russian). MR 760999

[4] R. Bos, Geometric quantization of Hamiltonian actions of Lie algebroids and Lie groupoids, Int. J. Geom. Methods Mod. Phys. 4 (2007), no. 3, 389-436. MR 2343354

[5] J. Cheeger and J. Simons, Differential characters and geometric invariants, Geometry and topology, LNM 1167, 1985, pp. 50-80. MR 827262

[6] K. Gomi, Equivariant smooth Deligne cohomology, Osaka J. Math. 42 (2005), no. 2, 309-337, available at arXiv:math/0307373 MR 2147734

[7] V. Guillemin and S. Sternberg, Supersymmetry and equivariant de Rham theory, Springer, 1999. MR 1689252

[8] M. Hopkins and I. Singer, Quadratic functions in geometry, topology, and M-theory, J. Differential Geom. 70 (2005), no. 3, 329-452, available at arXiv:math/0211216 MR 2192936

[9] B. Kostant, Quantization and unitary representations. I. Prequantization, Lectures in modern analysis and applications, III, LNM 170, 1970, pp. 87-208. MR 0294568 
[10] E. Lerman and A. Malkin, Differential characters as stacks and prequantization, available at arXiv:0710.4340

[11] E. Lupercio and B. Uribe, Differential characters on orbifolds and string connections. I. Global quotients, Gromov-Witten theory of spin curves and orbifolds, Contemp. Math., vol. 403, 2006, pp. 127-142, available at math.DG/0311008 MR 2234887

[12] J. Marsden and A. Weinstein, Reduction of symplectic manifolds with symmetry, Rep. Mathematical Phys. 5 (1974), no. 1, 121-130. MR 0402819

[13] K. Meyer, Symmetries and integrals in mechanics, Dynamical systems, Academic Press, New York, 1973, pp. 259-272. MR 0331427

[14] I. Moerdijk and J. Mrčun, Introduction to foliations and Lie groupoids, Cambridge Studies in Advanced Mathematics, vol. 91, Cambridge University Press, Cambridge, 2003. MR 2012261

[15] A. Weil, Sur les théorèmes de de Rham, Comment. Math. Helv. 26 (1952), 119-145 (French). MR 0050280

[16] A. Weinstein, Symplectic groupoids and Poisson manifolds, Bull. Amer. Math. Soc. (N.S.) 16 (1987), no. 1, 101-104. MR 866024

Department of Mathematics, University of Illinois at Urbana-Champaign, 1409 W. Green Street, Urbana, IL 61801, USA 